\title{
XCVII. On the highest wave in deep water
}

\section{J.R. Wilton M.A. B.Sc.}

To cite this article: J.R. Wilton M.A. B.Sc. (1913) XCVII. On the highest wave in deep water, Philosophical Magazine Series 6, 26:156, 1053-1058, DOI: 10.1080/14786441308635056

To link to this article: http://dx.doi.org/10.1080/14786441308635056

册 Published online: 08 Apr 2009.

Submit your article to this journal 중

Џll Article views: 4

Q View related articles $\square$

4 Citing articles: 2 View citing articles $\square$ 
more than $3 \times 10^{1 \mathrm{l}} \mathrm{ohms}$; with such a resistance a change in the current of $3 \times 10^{-15}$ amp. can be certainly detected. If a higher resistance is taken the conductivity of the insulation of the apparatus is apt to be comparable with that of the resistance, and if, as is usually the ease, the currents measured are those due to radioactive rays, Schweidler's fluctuations prevent any increase in the accuracy of the measuroment *.

In conclusion it may be pointed out that these high resistances appear to provide the most convenient means of determining small currents of $10^{-10}$ amp. or less in absolute measure. They are both cheaper and easier to use than either standard capacities or quartz piezoelectrics. Perhaps some instrument-maker will undertake the construction of such standardized high resistances.

\section{Summary.}

Some further information is given as to the high resistances made of mixtures of xylol and alcohol contained in glass cells. The conclusions important for the practical construction of such resistances are given in $\S 5$.

Leeds University, Oct. 1913.

XCVII. On the Highest $W_{\text {ave }}$ in Deep Water. By J. R. WiLton, M.A., B.Sc., Assistant Lectirev' in Mathematics in the University of Sheffield $\dagger$.

T' is possible to obtain a fluid motion in two dimensions 1 with any given curve as free surface. (The "motion" spoken of in this paper is, throughout, to be understood to mean steady motion.)

Let the axis of $x$ be horizontal, that of $y$ vertically upwards. Let $\quad z=x+\iota y, \quad w=\dot{\phi}+\iota \psi$,

* It seems that some persons who have used such resistances (and also Bronson resistances) for the measurement of currents have estimated the current by earthing that end of the resistance which is not attached to the quadrant of the electrometer and observing to what potential the quadrant rises. A far more convenient, accurate, and rapid method is to connect that end of the resistance to a potentiometer and to observe to what potential that end must be raised in order that the electrometerneedle should not move when the quadrant is insulated disconnected from earth. The rapidity of the measurements is then independent of the time which the needle of the electrometer takes to come to rest. This method would appear specially suitable for observations of radionctive substances with a short period of decay. It must be noted that if, as usually happens, there is a small "zero F.M.F." (p. 1048), its effects can be eliminated, either by subtracting it from the potentiometer readings or, more conveniently, by changing the sign of the current. The zero E.MI.F. will be found to be wonderfully constant over days.

$\dagger$ Commuricated by the Author. 
where $\phi$ and $\psi$ are the velocity potential and the stream function, respectively.

Then the motion for which the free surface is given by eliminating $\theta$ between $x=\mathrm{X}(\theta), y=\mathrm{Y}(\theta)$ is represented by the equations,

$$
\left.\begin{array}{rl}
z & =\mathrm{X}(\theta)+\iota \mathrm{Y}(\theta), \\
w & =\int \sqrt{[\mathrm{C}-2 g \mathrm{Y}(\theta)]\left(\mathrm{X}^{\prime 2}+\mathrm{Y}^{\prime 2}\right)} d \theta,
\end{array}\right\},
$$

where $\theta$ is a complex variable, $\mathrm{X}$ and $\mathrm{Y}$ are functions of $\theta$ which are real when $\theta$ is real, and $X^{\prime}$ and $Y^{\prime}$ denote rospectively $\frac{d \mathrm{X}}{d \theta}$ and $\frac{d \mathrm{Y}}{d \theta}$.

For the condition to be satisfied on the free surface, $\psi=0$, is

$$
q^{2}=\mathrm{C}-2 g y,
$$

where $q$ is the resultant velocity, $i$. $e$.

$$
q^{2}=\left|\frac{d x}{d z}\right|^{2}={ }_{j}^{\prime}(\mathrm{C}-2 g \mathrm{Y})\left(\mathrm{X}^{\prime 2}+\mathrm{Y}^{\prime 2}\right)\left(\frac{d \theta}{d z}\right)^{2} \mid
$$

But $\psi=0$ if $\theta$ is real (provided $\mathrm{C}-2 g \mathrm{Y}$ is positive),

i. e.

$$
\begin{aligned}
q^{2} & =\mid(\mathrm{C}-2 g \mathrm{Y}) \frac{\mathrm{X}^{\prime}-\imath \mathrm{Y}^{\prime}}{\mathrm{X}^{\prime}+\imath \mathrm{Y}^{\prime}}=\mathrm{C}-2 g \mathrm{Y} \\
& =\mathrm{C}-2 g y,
\end{aligned}
$$

for on the surface, since $\theta$ is real,

$$
x=\mathrm{X}(\theta), \quad y=\mathrm{Y}(\theta) .
$$

The equation of the free surface may thus be chosen arbitrarily. But the converse problem of determining the form of the free surface when some other condition is given can only be attacked by tentative methods.

It will be convenient for our purpose to put $\theta=\mathrm{F}(w)$, and $\mathrm{Y}(\theta)=\theta$. This does not in any way limit the generality of the result. We then have

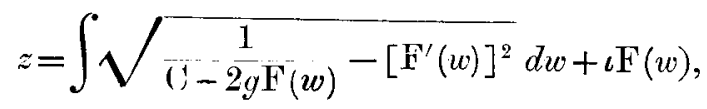

and the equation of the free surface is

$$
\begin{aligned}
y & =\mathrm{F}(\phi), \\
x & =\int \sqrt{\frac{1}{\mathrm{C}-2 g \mathrm{~F}(\phi)}-\left[\mathrm{F}^{\prime}(\phi)\right]^{2}} d \phi \\
& =\int \sqrt{\frac{1}{(\mathrm{C}-2 g y)\left(\frac{d y}{d \phi}\right)^{2}-1} d y .}
\end{aligned}
$$


The function $F(\phi)$ must be real. There must also be a limit to the form of $\mathrm{F}$ owing to the fact that $x$ must be real.

If we suppose that the depth of the fluid is infinite, the condition to be satisfied is

$$
\frac{d z}{d w}={ }_{c}^{1}, \text { when } \psi=-\infty \text {. }
$$

A function satisfying these conditions is that given by the equation

$$
\frac{g w}{c^{3}}=\sin ^{-1} \sqrt{\frac{2 g \mathrm{~F}}{c^{2}}}+\sqrt{\frac{2 g \mathrm{~F}}{c^{2}}\left(1-\frac{2 g \mathrm{~F}}{c^{2}}\right)},
$$

for which

$$
z=\sqrt{\mathrm{F}}\left(\frac{2 c^{2}}{g}-\mathrm{F}\right)+\frac{2 c^{2}}{g} \sin ^{-1} \sqrt{\frac{\overline{g F}}{2 c^{2}}}+\iota \mathrm{F},
$$

where in applying equation (1) we have put $\mathrm{C}=c^{2}$; and the equation of the free surface is

$$
x=\sqrt{y\left(\frac{2 c^{2}}{g}-y\right)}+\frac{2 c^{2}}{g} \sin ^{-1} \sqrt{\frac{g y}{2 c^{2}}} . .
$$

Since $y=\mathrm{F}$, and has to be real, the greatest value of $y$ is $c^{2} / 2 g$, and the least is 0 . Therefore the amplitude of the wave is $c^{2} / 2 g$, which is the value of $y$ at the crest.

The wave-length is

$$
\begin{aligned}
\lambda & =2\left[\sqrt{y}\left(\frac{2 c^{2}}{g}-y\right)+\frac{2 c^{2}}{g} \sin ^{-1} \sqrt{\frac{g y}{2 c^{2}}}\right]_{0}^{\frac{c^{2}}{2 g}} \\
& =2 \frac{c^{2}}{2 g}\left(\sqrt{3}+\frac{2 \pi}{3}\right) \\
& =\left(2 \sqrt{ } 3+\frac{4 \pi}{3}\right) a \\
& =7 \cdot 6 a, \text { nearly }
\end{aligned}
$$

where $a$ is the amplitude

The velocity is given by

$$
\frac{2 \pi c^{2}}{g \lambda}=\frac{4 \pi \alpha}{\lambda}=\frac{3}{1+\frac{3 \sqrt{ } \cdot 3}{2 \pi}}=1 \cdot 64
$$

At the crest, where $y=\frac{c^{2}}{2 g}$, we find

$$
\frac{d x}{d y}= \pm \sqrt{ } 3
$$

so that there is a sharp angle of $120^{\circ}$ in the wave-profile at this point. 
This result is in agreement with the work of Stokes and of Michell on the "highest wave" in water. The ratio of wave-length to amplitude is also in fair agreement with the approximate value given by Michell*, but the velocity is considerably greater. He gives

$$
\text { and } \quad c^{2}=\cdot 191 g \lambda \text {, or } \quad \frac{2 \pi c^{2}}{g \lambda}=1 \cdot 20 \text {. }
$$

Michell's series appear to converge fairly rapidly, but the addition of terms of higher order would certainly tend to increase his estimate of the velocity.

The wave-profile evidently consists of a succession of ares of cycloids which meet one another at an angle of $120^{\circ}$. If we put $y=\frac{2 c^{2}}{g} \sin ^{2} \frac{\theta}{2}$, the equation of the free surface takes
the form

$$
\left.\begin{array}{l}
r=\frac{c^{2}}{g}(\theta+\sin \theta) \\
y=\frac{c^{2}}{g}(1-\cos \theta)
\end{array}\right\}, \cdot .
$$

where the whole wave-length is included between $\theta=-\pi / 3$ and $\theta=\pi / 3$. Certain easily determinable constants of integration must be added to the expression for $x$ in the various cases when $\theta$ has any other of its possible ranges of values on the free surface:- $-e . g$. when $\frac{5 \pi}{3}<\theta<\frac{7 \pi}{3}$ the constant is $\frac{e^{2}}{g}\left(\frac{4 \pi}{3}-\sqrt{ } 3\right)$.

We may verify this result by determining the steady motion for which equations (3) represent a free surface. It will be given by

whence

$$
\begin{aligned}
z & =\frac{c^{2}}{g}[\theta+\sin \theta+\iota(1-\cos \theta)] \\
w & =\frac{c^{2}}{g} \int \sqrt{\left[c^{2}-2 c^{2}(1-\cos \theta)\right] 2(1+\cos \theta)} d \theta, \\
& =\frac{2 e^{3}}{g} \int \sqrt{2 \cos \theta-1} \cos \frac{\theta}{2} d \theta
\end{aligned}
$$

$$
\frac{g w}{2 c^{3}}=\sin \frac{\theta}{2} \sqrt{1-4 \sin ^{2} \frac{\theta}{2}}+\frac{1}{2} \sin ^{-1}\left(2 \sin \frac{\theta}{2}\right) .
$$

* Phil. Mag. Norember 1893. 
We may write the equation for $z$ in the form

$$
\frac{g z}{c^{2}}=\theta+\iota-\iota e^{\imath \theta} \text {. }
$$

Then

This gives

$$
\begin{aligned}
\frac{d w}{d z} & =\frac{2 c^{3}}{g} \sqrt{2 \cos \theta-1} \cos \frac{\theta}{2} \frac{d \theta}{d z} \\
& =c \sqrt{1-e^{-\imath \theta}+e^{-22 \theta}} .
\end{aligned}
$$

i.e. $\quad q^{2}=c^{2}-2 g y$ on the surface.

Further, the bottom of the liquid corresponds to $\theta=-\infty$ $(y=-\infty, \psi=-\infty)$, which makes

$$
\frac{d w}{d z}=c
$$

as it should be.

Hence equations (3), where it is understood that

$$
\frac{1}{2}<\cos \theta<1 \text {, }
$$

and that, when $\theta$ does not lie between $\frac{-1}{3}$ and $\frac{1}{3}$, the appropriate constant is to be added to $x$ in order to make the ares of the various cycloids "fit on," represent a possible form of the "highest wave" in deep water.

The necessity of "fitting on" the different arcs of the cycloid leads to a difficulty which cannot satisfactorily be overcome. There is a discontinuity in the motion across the vertical lines through the crests of the wave, for the vertical velocity does not vanish on these lines, except at the crest of the wave and at the bottom of the fluid; and, moreover, its direction, but not its magnitude, is changed in passing across these lines. This necessitates a constant change of momentum in passing across these lines, which change, it will be seen, is vertically upwards, so that it can be supplied, and the above equations will therefore represent an exact solution of the problem, if we suppose that the bottom of the fluid is a rigid horizontal plane.

The solution is, however, more interesting when regarded as an approximation to the actual physical solution of the problem of determining the form of the highest wave in deep water. That it can be so regarded is due to the fact that along the vertical lines through the crests $v$ is small. I find, for $x=\frac{c^{2}}{g}\left(\frac{\pi}{3}+\frac{\sqrt{ } 3}{2}\right)$, the following table connecting $u$ and $v$ with $y:-$ 


\begin{tabular}{|c|c|c|}
\hline$g \underline{y}$ & $\underline{u}$ & $\underline{v}$ \\
\hline & $c^{\circ}$ & $c^{\circ}$ \\
\hline$-\infty$ & 1 & 0 \\
\hline$-4 \cdot 82$ & $\cdot 912$ & -.024 \\
\hline$-2 \cdot 43$ & $\cdot 869$ & $-.03 !$ \\
\hline-59 & $\cdot 747$ & $-\cdot 034$ \\
\hline$-\cdot 18$ & $\cdot 658$ & -.023 \\
\hline$+\cdot 18$ & $\cdot 505$ & $-\cdot 010$ \\
\hline$+\cdot 5$ & 0 & 0 \\
\hline
\end{tabular}

The negative sign shows that the direction of $v$ is downward.

XCVIII. Intelligence and Miscellaneous Articles. ON THE EXISTENCE OF URANIUM Y. Gentlemen, T'o the Editors of the Philosophical Magazine. TWO years ago I gave an account in this Journal (Phil. Mag. xxii. p. 419, 1911) of experiments which indicated the presence of a new product in uranium of half period of transformation 1.5 days, which I called uranium $Y$. Evidence was given that this new radioactive substance must be regarded as a branch product of the main uranium series. In the May number of this Journal Mr. A. Fleck described some experiments in which he was unable to separate this new product, and concluded that the results obtained by me were possibly to be ascribed not to a new product but to the presence of thorium in my uranium preparations. In my reply (Phil. Mag. Aug. 1913) I gave reasons for doubting the correctness of Mr. Fleck's conclusions, and expressed the view that it was impossible to explain my results by the presence of thorium products, as the radioactive constants of uranium $\mathrm{Y}$ were quite different from those to be expected for the products of thorium.

In his experiments $\mathrm{Mr}$. Fleck employed a highly purified uranium salt which had been prepared some years before by $\mathrm{Mr}$. F. Soddy, F.R.S. It seems possible that the inability of Mr. Fleck to find evidence of uranium $Y$ might be due to the removal of its parent substance in the methods of purification. In order to throw light on this point, Mr. Soddy kindly loaned to me about 60 grammes of his pure uranium nitrate. I repeated my original experiments with this material, and was at once able to separate uranium $\bar{Y}$ from it.

Since Mr. Fleck was unable to repeat my work, I am intending to publish shortly a paper giving a more detailed account of my experimental methods. I trust that other investigators will then find no difficulty in repeating my original experiments.

I am at present making further investigations to determine more accurately the ratios of the activities of uranium $X$ and uranium $\mathrm{Y}$, comparing the penetrating rays of uravium $\mathrm{X}$ with the most penetrating rays from uranium $Y$. I have found that the activity of the latter must be more than 2 per cent. of the former. This ratio is of the order of magnitude to be expected if the actinium series branched off at this point. G. N. Antonofr.

Chemical Laboratory of the Academy of Sciences, St. Petersburg. 